\title{
Autoconceito, educação física e alunos de 6 a 10 anos: uma revisão sistemática da literatura
}

\author{
Self-concern, physical education and students from 6 to 10 years: \\ a systematic review of the literature
}

DOI: $\underline{\text { http://dx.doi.org/10.36453/2318-5104.2019.v17.n2.p111 }}$

\author{
Geisan Munique Giovanetti Gomes ${ }^{1}$, Fábio Ricardo Hilgenberg Gomes², \\ Paulo Sérgio Ribeiro ${ }^{1}$ \\ ${ }^{1}$ Universidade Estadual de Ponta Grossa (UEPG) \\ ${ }^{2}$ Universidade Federal do Paraná (UFPR)
}

RESUMO

Objetivo: Revisar a relação do Autoconceito com a Educação Física em alunos de 6 a 10 anos. Método: A busca dos descritores foi realizada em quatro base de dados (Redalyc, BVS, Scielo e Science Direct). O período de buscas da publicação dos manuscritos compreendeu de janeiro de 2013 a maio 2018. A avaliação da qualidade dos estudos foi realizara através dos checklist STROBE e DOWNS e BLACK. Resultados: foram encontrados na busca de títulos 257 estudos, foram lidos 197 resumos e selecionados 5 artigos para leitura na integra (seguindo os fatores de inclusão e exclusão), estes foram incluídos na revisão com os desfechos necessários para a análise. Conclusão: A Educação Física escolar mostrou-se um fator positivo perante o construto do autoconceito em crianças de 6 a 10 anos, porem em relação a está faixa etária a literatura ainda se mostra limitada, necessitando de mais investigações na área.

PALAVRAS-CHAVE: Autoconceito; Educação Física; Crianças.

\section{ABSTRACT}

Objective: This study aimed to systematically review the relationship between self-concept and physical education in students aged 6 to 10 years. Method: The search for descriptors was carried out in four databases (Redalyc, BVS, Scielo and Science Direct). The period of search of the manuscript publication comprised from January 2013 to May 2018. The quality evaluation of the studies was performed through the checklists STROBE and DOWNS and BLACK. Results: 257 studies were found in the search for titles, 197 abstracts were read and 5 articles were selected for reading comprehensively (following the inclusion factors and exclusion), these were included in the review with the necessary outcomes for the analysis. Conclusion: School Physical Education was a positive factor in the construction of self-concept in children from 6 to 10 years of age, but in relation to this age group, literature is still limited, requiring more research in the area.

KEYWORDS: Self-concept; Physical Education; Children. 


\section{INTRODUÇÃO}

A educação física escolar mostra-se como uma importante disciplina para o desenvolvimento do aluno através das atividades realizadas em sua prática, as quais podem desenvolver valores necessários para a sua formação (SANTOS; BRANDÃO, 2018). Está área do conhecimento humano ligada as práticas corporais mostram-se indispensáveis para as crianças, influenciando no desenvolvimento de seu autoconceito, e na construção de dimensões importantes no decorrer de sua vida, como a saúde e processos mentais (SERASSUELO JUNIOR et al., 2012).

$\mathrm{O}$ autoconceito pode ser definido como a forma que o indivíduo tem de perceber a sí mesmo, pois “... pressupõe uma integração de percepções que se referem às características, atributos, capacidades, limites, valores, relações que o sujeito reconhece como descritivos de si próprio e que entende como integrados na sua identidade" (TAGARRO, 2012, p. 2). De acordo com o exposto o presente trabalho torna-se relevante em buscar na literatura atual a discussão sobre 0 autoconceito e de como a educação física pode contribuir neste construto, principalmente em crianças de 6 a 10 anos, pois "Há um consenso na literatura de que o autoconceito começa a ser construído na infância" (SUEHIRO et al., 2009, p. 3). Através desta revisão sistemática será possível contribuir para a formação dos docentes, corroborando para o entendimento da Educação Física Escolar e atividades que atendam a melhora do autoconceito em crianças, expondo a visão mais crítica da ciência no ambiente escolar.

O autoconceito está relacionado com o desempenho acadêmico, de forma que este construto se mostra um importante fator no desenvolvimento da criança em seu processo de aprendizagem (GASPAROTTO, 2018; KLAPP, 2017). O desenvolvimento da criança é marcado por fases e alterações comportamentais, os quais podem corroborar para o desenvolvimento de suas percepções de competências em relação ao seu ambiente escolar e social (GALLAHUE; OZMUN, 2008). Cada criança condiz com a vivência de cada gênero, seja na escola ou com a família e a sociedade em que o cerca, portanto, a escola tende a ser participativa com a comunidade realizando a construção do seu currículo baseado na percepção de comunidade escolar e aliando ao construto do autoconceito (GASPAROTTO, 2018, p. 10).

Alguns estudos apontam para o pressuposto de que a atividade física influncia na vida da criança, afetando no desenvolvimento de seu autoconceito (TAGARRO; VIEGA, 2012; BRAUNER; VALENTINI; SOUZA, 2017; SOUZA; SPESSATO; VALENTINI, 2014). Assim como a Educação Física vem buscando mostrar sua identidade no ambiente escolar, a qual demostra ser eficiente em diversos fatores cognitivos, entre eles a emocionalidade do aluno (SERASSUELO JUNIOR et al., 2012). De acordo com o exposto acima, a presente pesquisa tem como objetivo revisar sistematicamente a literatura em relação do Autoconceito com a Educação Física em alunos de 6 a 10 anos no período de janeiro 2013 até maio de 2018.

\section{MÉTODOS}

Revisão Sistemática são estudos secundários que reúnem estudos semelhantes publicados, os quais são selecionados, revisados e avaliados criticamente em sua metodologia, reunindo numa análise estatística e metanálise quando isto é possível, na perspectiva de sintetizar estudos primários semelhantes e de boa qualidade (SAMPAIO; MANCINI, 2007).

A identificação dos artigos relevantes para esta revisão foi realizada por meio de busca em quatro bases de dados eletrônicas: Scielo, Science Directy, Redalyc e BVS. As buscas de artigos foi limitada ao período de janeiro de 2013 a maio de 2018, considerando artigos publicados nos idiomas português, inglês e espanhol. Foram utilizados descritores caracterizando componentes de Autoconceito, Educação Física e Crianças de 6 a 10 anos de acordo com os descritores das ciências da saúde (DeCS). A busca dos estudos foram feitas com descritores em língua inglesa, espanhola e portuguesa e as combinações realizadas entre os descritores mediante a utilização dos operadores booleanos "AND" e "OR". Optou-se por não incluir teses, dissertações, estudos de revisão e monografias.

Uma análise inicial foi realizada com base nos títulos dos manuscritos, seguido da avaliação nos resumos de todos os artigos que preenchiam os critérios de inclusão ou que não permitiam se ter certeza de que deveriam ser excluídos. Após análise dos resumos, todos os artigos selecionados foram obtidos na íntegra e posteriormente examinados de acordo com os critérios de inclusão estabelecidos. Também foi realizada uma busca manual em listas de referências dos artigos selecionados. Foram considerados os seguintes critérios de inclusão: (i) artigos originais publicados em periódicos peer-reviewed que tiverem objetivo de verificar a relação ou associação entre Autoconceito, Educação Física e Crianças de 6 a 10 anos (ii) estudos publicados entre janeiro de 2013 e maio de 2018; e (iii) amostras com crianças em idade escolar de 6 a 10 anos. Estudos de intervenção, transversais e longitudinais serão considerados nessa revisão. Os critérios de exclusão adotados foram: (i) crianças abaixo de 6 anos e acima de 10 anos. (ii) teses, monografias, revisões sistemáticas e disssertações. (iii) foram excluídos estudos publicados abaixo de janeiro de 2013.

Dois revisores independentes avaliaram a qualidade dos estudos utilizando o checklist traduzido para o Brasil por 
Malta et al. (2010). Este checklist é utilizado para verificar a qualidade de estudos transversais "Strengthening the Reporting of Observational Studies in Epidemiology (STROBE)", para avaliação da qualidade dos artigos com esses delineamentos. A lista de checagem do STROBE contém vinte e dois itens, com recomendações sobre o que deverá ser incluído em uma descrição mais precisa e completa de estudos observacionais. Todas as questões foram codificadas como 0 (representando qualidade inadequada) ou 1 (representando qualidade adequada).

Portanto, os escores de qualidade do estudo variou de 0 a 22 pontos nos estudos. Para estudos de intervenção foi utilizado o checklist Downs e Black (1998), que consiste em vinte e sete itens, com recomendações sobre o que deverá ser incluído em uma descrição mais precisa e completa de estudos de intervenção. Todas as questões foram codificadas como 0 (representando qualidade inadequada) ou 1 (representando qualidade adequada). Portanto, os escores de qualidade dos estudos variou de 0 a 27 pontos nos estudos. No caso de eventuais diferenças entre os dois revisores na avaliação dos artigos, foram realizadas reavaliações do artigo até ambos os revisores concordarem com a avaliação, sendo considerado ponto de corte de 17 pontos para o STROBE e de 20 pontos para o Downs e Black (1998), pois os estudos que não atingiram a pontuação de corte não foram considerados nesta revisão.

Para os estudos que foram incluídos nesta revisão, os seguintes dados foram extraídos: Título/Autor-ano/Qualidade dos estudos (DOWS e BLACK ou STROBE), tipo e tamanho da amostra, variáveis investigadas, objetivo geral, tipo de estudo e os principais resultados. Os artigos foram organizados em ordem cronológica considerando o ano de publicação do estudo.

\section{RESULTADOS}

A Figura 1 apresenta o fluxograma descrevendo o processo de busca e seleção dos estudos. Foram identificados 257 artigos potencialmente relevantes para esta revisão. Após a análise dos títulos, 60 estudos foram selecionados para leitura dos resumos. Treze estudos foram considerados relevantes na leitura do resumo, sendo selecionados para leitura do texto na íntegra. Destes, 8 estudos $(61,5 \%)$ foram excluídos por não atenderem os critérios de elegibilidade, sendo que 3 estudos não apresentaram uma ou mais variáveis; 2 estudos estavam duplicados; e 3 estudos tratavam de adolescentes. Portanto, a busca eletrônica gerou 5 estudos relevantes para essa revisão sistemática. Após a leitura das referências dos artigos selecionados, nenhum estudo apresentou os critérios de inclusão para fazerem parte nesta revisão.

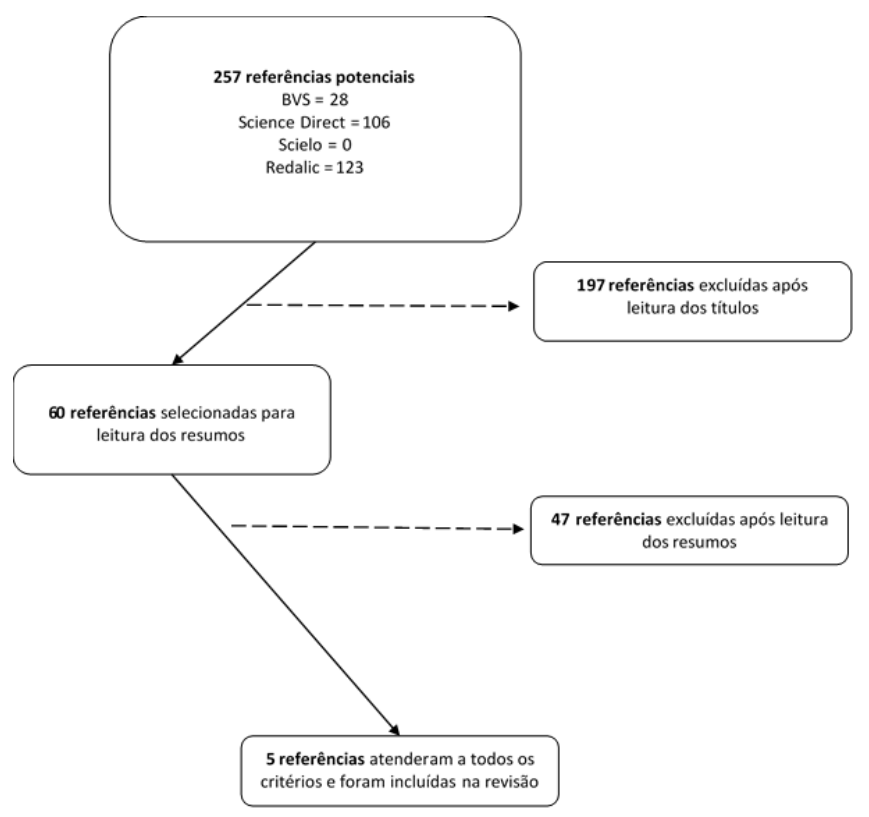

Figura 1. Fluxograma de busca e seleção dos estudos nesta revisão.

A pontuação dos estudos de intervenção variou de 21 a 23 pontos, sendo que dois alcançaram a pontuação de 23 pontos, um com 22 pontos e um com 21 pontos. O estudo transversal obteve a pontuação de 20 pontos. Todas as características e os principais resultados dos estudos revisados podem ser observados detalhadamente no Quadro 1 dessa revisão. Foram encontrados quatro estudos de intervenção e um estudo transversal. 


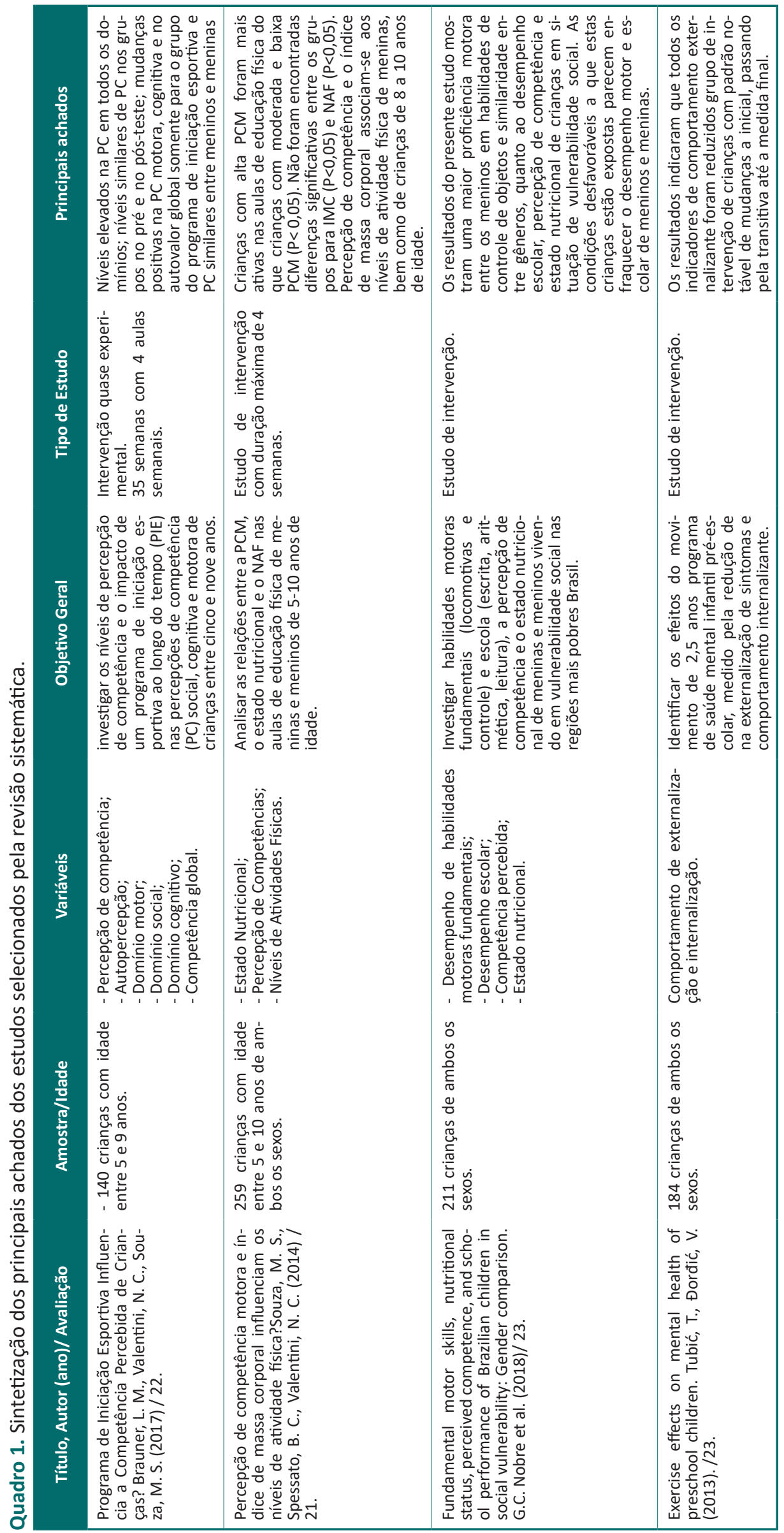


Dos estudos, três (60\%) foram realizados no Brasil, um (20\%) na Espanha, e um (20\%) na Sérvia. Com relação aos estudos pesquisados, esses dados ajudam a observar que o Brasil tem maior preocupação com o autoconceito relacionado à educação física escolar na idade de 6 a 10 anos. Todos os estudos (100\%) envolveram crianças de ambos os gêneros. Das amostras inicialmente tratadas nos artigos, (100\%) foram realizadas em escolas. De acordo com as amostras dos artigos o autoconceito foi avaliado através das variáveis: Percepção de Competência, Autopercepção, Domínio Cognitivo e Competência Global. A avaliação do autoconceito também procedeu através de Atividades Físicas (Domínio Motor), ou seja, a Autopercepção das crianças por meio da Competência Motora.

Os estudos utilizaram os seguintes instrumentos: Pictorial Scale of Perceived Competence and Social Acceptance (PSPCSA), Escala de Autopercepção (EAP) , de Harter (1985) para crianças alfabetizadas, Desempenho escolar, Competência percebida, o Teste de Desempenho Escolar - SPT (STEIN, 1994), o Perfil de Autopercepção da Criança - SPPC (HARTER, 2012a, 1982; VALENTINI et al., 2010), e o Questionário de Comportamento Aberrante (HOŠEK; MOMIROVIĆ; BALA, 2003). Porém, o estudo espanhol avaliou o autoconceito de forma qualitativa através de desenho. $O$ desenho infantil pode promover a compreensão do brincar das crianças, a fim de mostrar alternativas, aqueles modelos de cultura física dominante impostos desde a primeira infância. A autopercepção de cada criança condiz com a vivência de cada gênero, seja na escola ou com a família e a sociedade em que a cerca.

O estudo espanhol e os três estudos brasileiros utilizaram de instrumentos relacionados a atividade física para a avaliação do domínio motor e/ou competência motora, verificando níveis de atividades físicas, Desempenho de habilidades motoras fundamentais, estado nutricional, tipo de jogo e domínio de atividade física. Mediante a essas variáveis, foi utilizado diversos instrumentos como: a percepção de competência motora de crianças não alfabetizadas, domínio motor da Escala Visual de Percepção de Competência, Aceitação para Crianças Jovens, Escala de Autopercepção para Crianças (EAPC), o Índice de Massa Corporal (IMC), as curvas do Center of Desease Control (CDC), teste de Desenvolvimento Motor Bruto, segunda edição - TGMD-2 (URICH, 2000).

O presente estudo buscou revisar sistematicamente as informações sobre o Autoconceito, Educação Física e Crianças de 6 a 10 anos. A busca rendeu poucos títulos, o que nos mostra a necessidade de estudos que envolvam essas variáveis.

Os estudos observaram que:

o autocoenceito incorpora, além de crenças percebidas sobre a competência individual em situações específicas, crenças de valor sobre si mesmo. Embora o construto autoconceito seja explorado por diversos enfoques psicológicos, alguns aspectos carecem ainda de melhor esclarecimento (SUEHIRO et al., 2009, p. 2).

Assim como o autoconceito pode ser correlacionado com a criatividade do aluno como no estudo de Tagarro e Veiga (2012, p. 5):

vários fatores que poderão influenciar o desenvolvimento ou inibição da criatividade no sujeito, será importante o foco nas suas características pessoais com vista a uma melhor compreensão da mesma. O autoconceito e a autoestima parecem exercer uma influência importante no desenvolvimento e manifestação da criatividade.

A Educação Física tem presença considerável em relação ao autoconceito, pois a maioria dos estudos analisados são realizados no horário escolar nas participações dos alunos nas aulas, onde ocorrem as avaliações das Percepções de Competências, análises de Atividades Físicas e motoras.

Segundo Souza, Spessato e Valentini (2014, p. 2), "[...] a escola e as aulas de educação física se destacam como meios de promoção de atividade física, uma vez que as crianças passam um longo período do seu dia na escola". As crianças de 6 a 10 anos de idade estão na transição da fase motora fundamental - estágio maduro para a fase especializada - estagio transitório (GALLAHUE; OZMUN, 2005; GALLAHUE; DONNELLY, 2008). Esse período transitório, na qual as crianças estão envolvidas ativamente na exploração e experimntação das capacidades motoras e passando para o período em que as habilidades estabilizadoras, locomotoras e manipulativas fundamentais são refinadas gradativamente. Como no estudo de Alcañiz e Bello (2016):

Os desenhos das crianças representam esportes e atividades competitivas em espaços ao ar livre na companhia de crianças. Pelo contrário, os desenhos das meninas representam atividades sedentárias ou de baixa intensidade realizada sob o teto na companhia de meninas e mulheres adultas. O desenho infantil pode promover a compreensão do brincar das crianças.

Então, nesta idade (6 a 10 anos) que as crianças fazem a relação de seu autoconceito e/ou autopercepção, ou seja, as formas como elas se veem nos aspectos pessoais e coletivos. $O$ esporte através das aulas de educação física, colabora na formação do aluno em relação a sua autopercepção e conhecimento de si mesmo pelo convívio social e afetivo com os demais alunos (ABI-EÇAB, 2017; SÁ, 2017). 
Este estudo apresenta algumas limitações. A primeira foi o uso de apenas quatro bases de dados, onde o número de estudos poderia ser maior se ocorressem em mais bases revisadas. Outra limitação é não incluir estudos com os mesmos descritores em adolescentes, pois a maioria dos estudos encontrados investigaram indivíduos acima de 12 anos e jovens. Contudo, o estudo mostra-se relevante, pois identificou poucos trabalhos, o que reflete a necessidade da realização de mais estudos nessa área com crianças de 6 a 10 anos, para aprimorar o conhecimento dos docentes.

\section{CONSIDERAÇÕES FINAIS}

Este estudo revisou sistematicamente a relação do autoconceito com a Educação Física em alunos de 6 a 10 anos. A leitura sobre os descritores autoconceito, educação física e crianças de 6 a 10 anos, são basicamente compostos por estudos de intervenção. Nesta busca foram encontrados apenas cinco estudos relacionados aos descritores pesquisados com os fatores de inclusão e exclusão citados no trabalho.

As aulas de educação física parecem ser um importante fator para o autoconceito, competência motora e atividade física. É através dela e do convívio coletivo que o aluno se percebe individual e socialmente, também a relação do esporte como um todo favorecer e corroborar para tal acontecimento. Por fim, faz-se necessário a realização de mais estudos envolvendo autoconceito em relação a educação física escolar em alunos do ensino fundamental I, pois é nessa idade que as crianças constroem parte da sua autopercepção e o conhecimento de sí mesmo.

\section{REFERÊNCIAS}

ABI-EÇAB, A. A função social do esporte na construção identitária dos sujeitos. Serviço Social em Revista, Londrina, v. 19, n. 2, p. 45-62, 2017.

ALCAÑIZ, F. M., BELLO, B. M. Juegos de niñas y juegos de niños: Estudio sobre la representación del juego infantil a través del dibujo. Revista Educación, San Pedro, v. 40, n. 1, p. 1-17, 2016.

BRAUNER, L. M.; VALENTINI, N. C.; SOUZA, M. S. Programa de iniciação esportiva influencia a competência percebida de crianças? Psico-USF, Bragança Paulista, v. 22, n. 3, p. 527-39, 2017.

DOWNS, S. H.; BLACK, N. The feasibility of creating a checklist for the assessment of the methodological quality both of randomised and non-randomised studies of health care interventions. Journal of Epidemiology \& Community Health, London, v. 52, p. 377-84, 1998.

FERRAZ, O. L. Educação física escolar: conhecimento e especificidade a questão da pré-escola. Revista Paulista de Educação Física, São Paulo, Supl. 2, p. 16-22, 1996.

GALLAHUE, D. L.; DONNELLY, F. C. Educação física desenvolvimentista para todas as crianças. São Paulo: Phorte, 2008.

GALLAHUE, D. L.; OZMUN, J. C. Compreendendo o desenvolvimento motor: bebês, crianças, adolescentes e adultos. São Paulo: Phorte, 2005.

GASPAROTTO, G. S.; SZEREMETA, T. P., VAGETTI, G. C.; STOLTZ, T.; OLIVEIRA, V. O autoconceito de estudantes de ensino médio e sua relação com desempenho acadêmico: Uma revisão sistemática. Revista Portuguesa de Educação, Braga, v. 31, n. 1, p. 21-37, 2018.

SERASSUELO JUNIOR, H.; BACARIN, D. S.; RONQUE, E. R. V.; OLIVEIRA, S. R. S.; SIMÕES, A. C. A percepção do autoconceito e sua influência no desempenho motor em crianças e adolescentes. Revista de Educação Física, Maringá, v. 23, n. 1, p. 15-23, 2012.

KLAPP, A. Does academic and social self concept and motivation explain the effect of grading on students' achievement? European Journal of Psychology of Education, New York, v. 33, n. 2, p. 355-76, 2017.

MALTA, M.; CARDOSO, L. O.; BASTOS, F. I.; MAGNANINI, M. M. F.; SILVA, C. M. F. P. Iniciativa STROBE: subsídios para a comunicação de estudos observacionais. Revista de Saúde Pública, São Paulo, v. 44, n. 3, p. 559-65, 2010.

NOBRE, G. C.; VALENTINIB, N. C.; NOBREA, F. S. S. fundamental motor skills, nutritional status, perceived competence, and school performance of brazilian children in social vulnerability: gender comparison. Child Abuse \& Neglect, Amsterdam, v. 80, p. 345, 2018.

SÁ, A. M. S. Motivação e aprendizagem: a influência familiar na vida escolar dos alunos da E. M. Raimundo Nonato Bogéa Ribeiro. Inter Espaço, Grajaú, v. 3, n. 11 p. 149-67, 2017.

SAMPAIO, R. F.; MANCINI, M. C. Estudos de revisão sistemática: um guia para síntese criteriosa da evidência 
científica. Revista Brasileira de Fisioterapia, São Carlos, v. 11, n. 1, p. 83-9, 2007.

SOUZA, M. S.; SPESSATO, B. C.; VALENTINI, N. C. Percepção de competência motora e índice de massa corporal influenciam os níveis de atividade física? Revista Brasileira de Ciência e Movimento, Brasília, v. 22, n. 2, p. 7886, 2014.

SUEHIRO, A. C. B. Avaliação do Autoconceito no Contexto Escolar: Análise das Publicações em Periódicos Brasileiros. Psicologia Ciência e Profissão, Brasília, v. 29 n. 1, p. 18-29, 2009.

TAGARRO, M.; VEIGA, F. H. Estudo da criatividade e do autoconceito em estudantes do ensino superior: Um projeto de investigação. In: II Seminário Internacional "Contributos da Psicologia em Contextos Educativos". Anais... Braga: Universidade do Minho, 2012.

TUBIĆ, T., ĐORĐIĆ, V. Exercise effects on mental health of preschool children. Anales de Psicología, Murcia, v. 29, n. 1, p. 249-56, 2013.

Autor correspondente: Geisan Munique Giovanetti Gomes

E-mail: geisan.munique@hotmail.com

Recebido: 12 de agosto de 2019.

Aceito: 01 de setembro de 2019. 